\title{
MR-Compatible Sectioning and Localization Device
}

National Cancer Institute

\section{Source}

National Cancer Institute. MR-Compatible Sectioning and Localization Device. NCI

Thesaurus. Code C148132.

A device designed to facilitate lesion detection and improve direct comparison of

histopathologic specimens with prior in vivo imaging, consisting of an MRI compatible localization device, with MR-visible fiducial marks for ex vivo imaging to facilitate radiog raphic-pathologic correlation. 